\title{
Cor triatriatum
}

\author{
L. K. LACQUET, J. A. LOS, J. NAUT A, A N D A. G . B R O \\ From the Departments of Thoracic Surgery and Anatomy-Embryology, University of Leyden, \\ The Netherlands
}

Cor triatriatum is a malformation of the heart in which the left atrium is divided into two compartments by an abnormal transverse or oblique septum. The postero-superior compartment receives the pulmonary veins, and the anteroinferior compartment comprises the left auricle and connects with the mitral orifice. The communication between the two compartments varies greatly.

True cor triatriatum is rare. In the necropsy material of 1,000 congenital heart defects, Abbott (1946) found only seven cases of cor triatriatum but 17 cases in which the left atrium contained abnormal adhesions or bands. Niwayama (1960) found four cases in 3,740 necropsies carried out from 1936 to 1958. Until a few years ago cor triatriatum was of interest only to the pathologist. But the number of clinical observations has increased since surgical correction has become possible. In 1952 Barnes and Finlay pointed out that this anomaly is amenable to surgical correstion. The first successful operations were performed by Lillehei and Lewis (1955), Vineberg and Gialloreto (1956), and Barrett and Hickie (1957).

We present four cases of cor triatriatum which received successful surgical treatment in our clinic. The anomaly was diagnosed pre-operatively in two cases. The third was operated upon for an atrial septal defect of the dorsal (secundum) type with mild mitral stenosis; the initial diagnosis in the fourth case was atrial septal defect.

\section{CASE REPORTS}

CASE 1 W. L. was a man aged 29 who in 1951 had had left-sided thoracic pain for a few weeks. Atrial fibrillation already existed at that time. After a motoring accident in 1958 he had a cough and heart palpitations with dyspnoea. In 1959 he was examined in the cardiology department because of dyspnoea with palpitations on the slightest exertion.

Clinical examination disclosed no oedema; the venous pressure was slightly increased. The heart rhythm was irregular, but no murmurs were audible.
The electrocardiogram revealed atrial fibrillation and a few isolated ventricular extrasystoles but no signs of right-sided or left-sided strain (Fig. 1). The chest radiograph showed a slightly increased heart volume with greatly broadened hila. The left atrium seemed slightly enlarged. The upper lung fields showed more pronounced vascularization than the lower fields. The left oblique projection disclosed an abnormal vascular shadow in the right upper field. The tomograms indicated that this opacity was probably a dilated pulmonary vein.

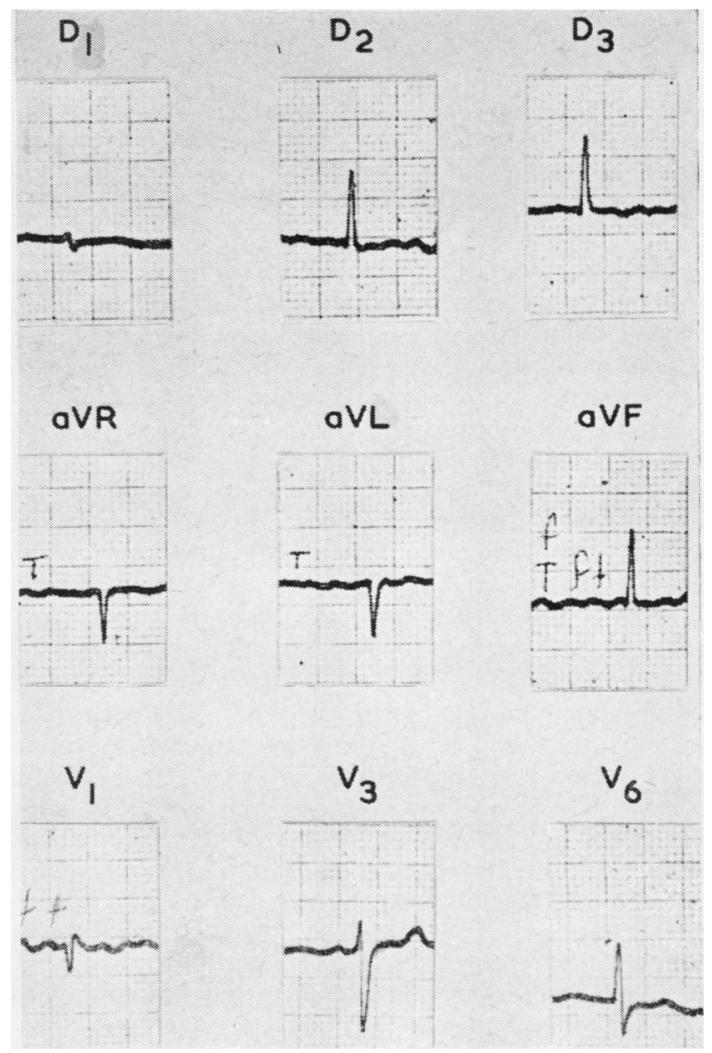

FIG. 1. Case 1. E.C.G. showing atrial fibrillation and isolated ventricular extrasystoles; no signs of left or right strain. 


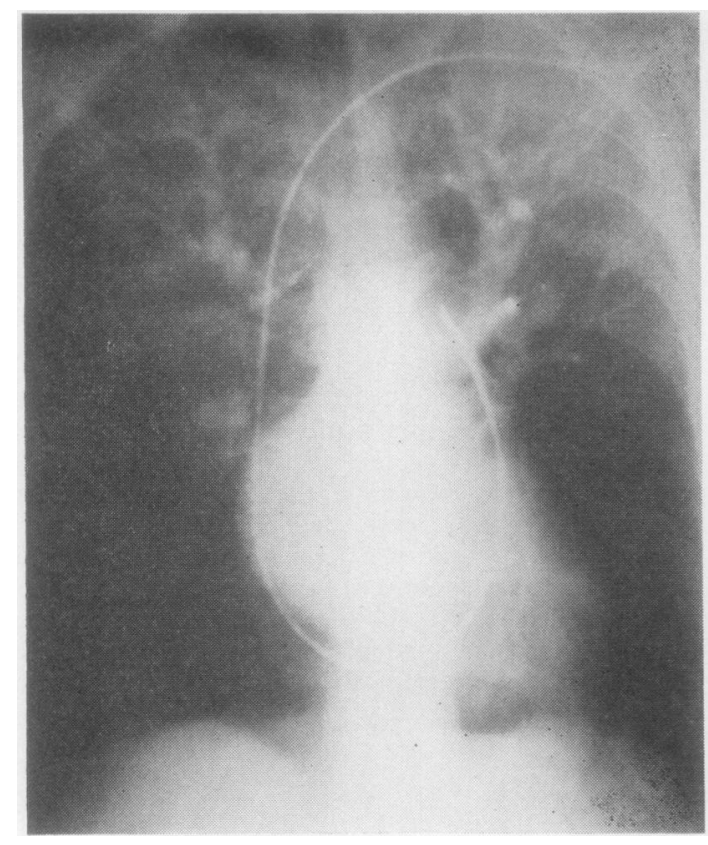

FIG. 2. Case 1. Angiocardiography in (left) antero-posterior and (right) lateral projections. The dilated pulmonary veins reach the left atrium via a distended compartment localized above the left atrium.

Angiocardiography revealed dilated and tortuous pulmonary veins reaching the left atrium via a distended compartment localized above the left atrium. These angiocardiographic features already suggested the existence of a cor triatriatum (Fig. 2).

At catheterization we found a pulmonary hypertension of $60 / 25 \mathrm{~mm}$. $\mathrm{Hg}$ and a mean wedge pressure of $26 \mathrm{~mm}$. $\mathrm{Hg}$. There were no shunts. Lung function tests showed a disturbed one-second forced expiratory volume $(38 \%)$ and an increased residue $(59 \%)$. Cor triatriatum was diagnosed.

On 7 June 1960 an operation was performed through an antero-lateral incision in the fourth left intercostal space. The entire left lower lobe was emphysematous. On palpation in the left atrium, no outlets of pulmonary veins were felt; towards the right, however, a protruding wall was felt in the left atrium. This wall contained a small orifice of about 0.5 sq. cm. (Fig. 3). A finger passed through this orifice (slightly tearing the wall) entered a space into which all the pulmonary veins opened. This was the spatium pulmonale. With the finger it was possible to open the septum completely, creating an opening of $6 \mathrm{sq}$. $\mathrm{cm}$. between the spatium pulmonale and the left atrium.

At the various clinical follow-ups the patient felt much better and less tired. Because of the pulmonary emphysema, however, the lung function remained unchanged.

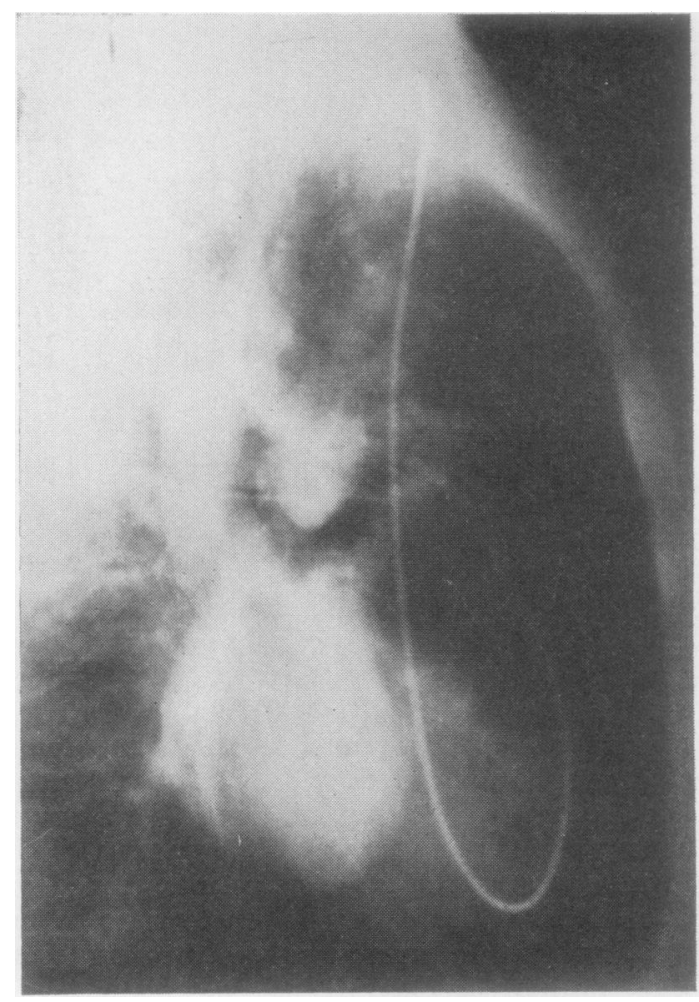

In this first case the diagnosis was made preoperatively on the basis of the angiocardiographic findings and the increased wedge pressure. The operation was carried out without hypothermia or extra-

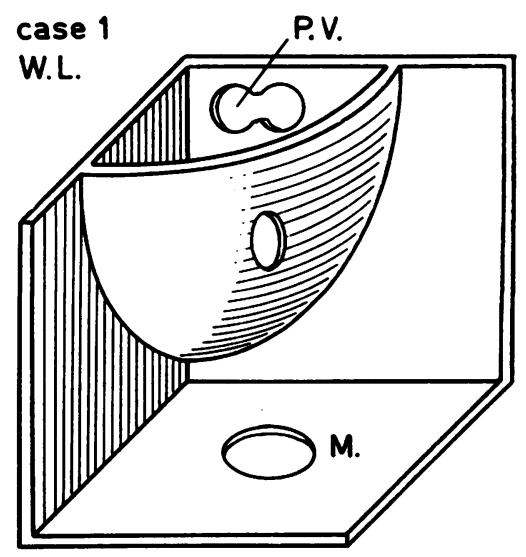

FIG. 3. Case 1. Diagram of the left atrium, divided into two compartments by an abnormal septum. The upper compartment receives the pulmonary veins $(P . V$.$) and the$ lower communicates with the mitral orifice (M.). The abnormal septum shows an orifice of $0.5 \mathrm{sq} . \mathrm{cm}$. 
corporeal circulation because the abnormal diaphragm could be digitally severed. In the abnormal septum there was an opening of $0.5 \mathrm{sq} . \mathrm{cm}$. between the third atrium and the left atrium. No communication existed between the third atrium and the right atrium.

CASE 2 V.Z. L. was an 11-year-old boy in whom a heart defect had been diagnosed in infancy. The boy had later had many respiratory infections and could walk only a few hundred yards. Examination in 1962 showed dyspnoea on the slightest exertion. Auscultation of the heart disclosed a grade 2 protosystolic souffle with its maximum on $4 \mathrm{~L}$ and at the apex. The liver was not palpable. The fingers and toes showed incipient turtle-back nails.

The electrocardiogram on 10 October 1960 showed a regular sinus rhythm and pronounced right ventricular hypertrophy. The phonocardiogram disclosed a holosystolic souffle on 4L (Fig. 4).

The antero-posterior chest radiograph taken on 11 October showed hardly any enlargement of the heart. The vascular pattern of the lungs showed unmistakable overfilling well into the periphery. The right atrium protruded slightly to the right, whereas the

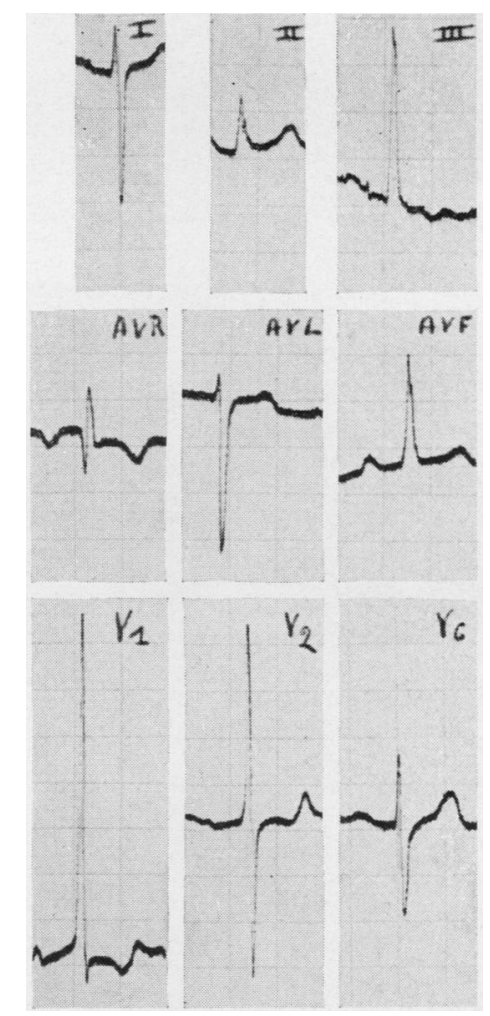

FIG. 4. Case 2. E.C.G. showing sinus rhythm; pronounced hypertrophy of the right ventricle. left atrium was small. The pulmonary arch was very prominent. The right oblique projection showed that the right ventricle extended as far as the anterior thoracic wall (Fig. 5).

Cineangiocardiography from the right ventricle indicated the existence of an accessory compartment in the right upper area into which the pulmonary veins opened. The pulmonary veins were dilated and remained filled for a long time.

At catheterization we found hypertension in the right ventricle $(82 / 2 \mathrm{~mm}$. $\mathrm{Hg})$ and in the pulmonary artery $(80 / 46 \mathrm{~mm}$. $\mathrm{Hg})$. Via an atrial septal defec: and via the third atrium we reached a pulmonary vein on the right with a pressure of $20 / 40 \mathrm{~mm}$. $\mathrm{Hg}$. When the catheter was withdrawn to the left atrium a pressure threshold was passed before the left atrium was entered.

The condition was diagnosed as cor triatriatum with dorsal (secundum) atrial septal defect.

An operation was performed on 17 October 1962 via an antero-lateral incision in the fifth right intercostal space, using extracorporeal circulation. In a retrocardiac position a compartment was felt which was clearly hypertensive and extended from right to left between the pulmonary veins of both lungs. The patient was connected to the heart-lung machine. The right atrium was opened and a small dorsal atrial septal defect of the central (secundum) type was found (Fig. 6). The atrial septum was cut open in the dorsal direction until the third atrium was reached. The orifice connecting the third atrium with the left atrium measured 0.5 sq. $\mathrm{cm}$. A right-to-left incision was then made in the tissue between the third atrium and the left atrium. As much of the tissue as possible was removed. The atrial septal defect and the atrial wall were then closed. Pressure readings no longer showed a gradient between the pulmonary veins and the left atrium.

The pulmonary hypertension necessitated a postoperative tracheotomy and respirator breathing for 24 hours. Thereafter this patient's course was uneventful.

At follow-up in December 1962 he was doing well and showed improved endurance. At a follow-up in November 1963 the boy was completely cured.

In this second case the cor triatriatum was likewise diagnosed pre-operatively on the basis of the cineangiographic findings, the hypertension in the pulmonary vein, and the pressure gradient between the third atrium and the left atrium. The operation was performed with extracorporeal circulation. An opening of $0.5 \mathrm{sq}$. $\mathrm{cm}$. existed between the third atrium and the left atrium. The left atrium communicated with the right atrium via a dorsal (secundum) atrial septal defect (Fig. 7).

CASE 3 C. U. was an 8-year-old girl. When examined in 1962 she had a two-year history of fatigue, dyspnoea on exertion, and pain in the chest. There was no cyanosis. Strong heart pulsations were visible and palpable on clinical examination. There was no 


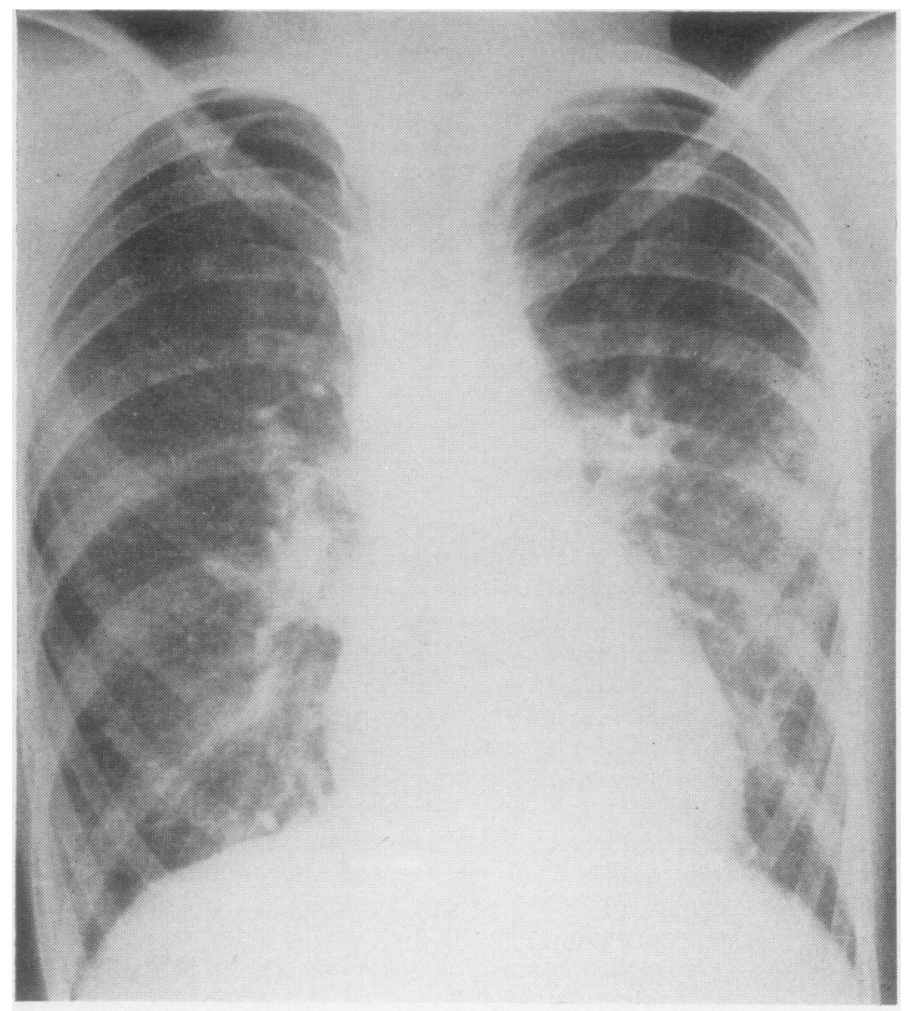

FIG. 5. Case 2. Chest radiographs in (a) antero-posterior projection: slightly enlarged right atrium and unmistakable overfilling of the lungs; prominent pulmonary arch; (b) right oblique projection: the right ventricle extends as far as the anterior thoracic wall.

thrill. Auscultation disclosed a grade 2 to 3 systolic murmur with a maximum on $4 \mathrm{~L}$ and a diastolic murmur at the apex and on $4 \mathrm{R}$ and $4 \mathrm{~L}$. The first sound at the apex was loud; the second sound on $2 \mathrm{~L}$ was inconspicuous and bifid. The liver was palpable $1 \mathrm{~cm}$. below the costal margin.

The electrocardiogram on 4 December 1962 disclosed a sinus rhythm, right axis deviation, right ventricular hypertrophy and strain, and right bundlebranch block (Fig. 8). The phonocardiogram revealed a soft, early systolic, decrescendo systolic souffle with a maximum on 2,3 , and $4 \mathrm{~L}$, and an early diastolic souffle on $4 R, L$, and over the apex. The duplicate second sound had a broad and loud second component. In the vectorcardiogram the frontal vector ran clockwise.

The chest radiograph taken on 21 December 1962 showed a greatly enlarged heart with a pronounced pulmonary vascular pattern. The right atrium and ventricle were enlarged, and the left atrium bulged (Fig. 9). The angiocardiogram from the right ventricle disclosed the large ventricle and large pulmonary artery, but the left atrium and ventricle were of normal size. Unmistakable recirculation was observed.

Heart catheterization showed a shunt at the atrial

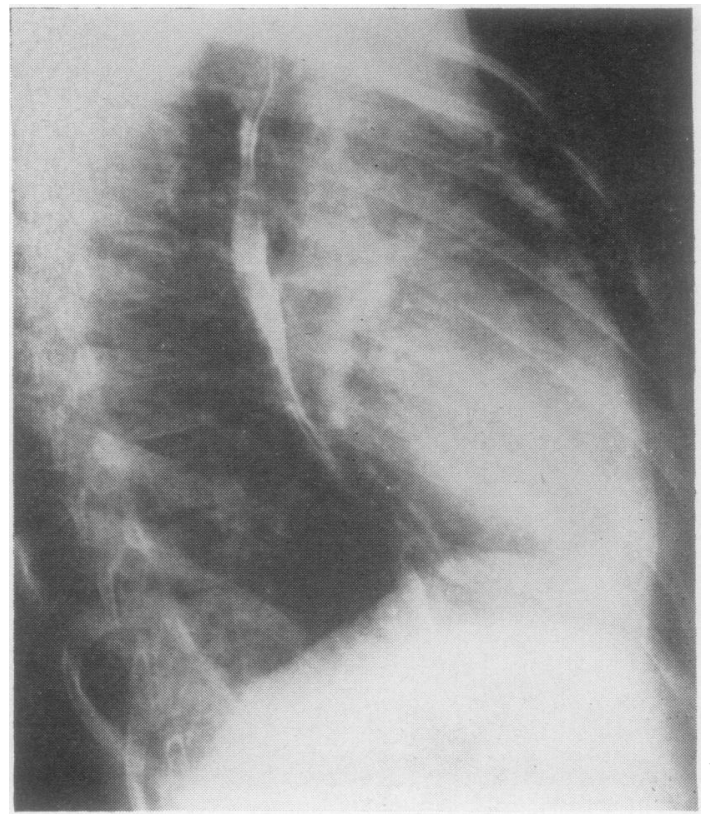




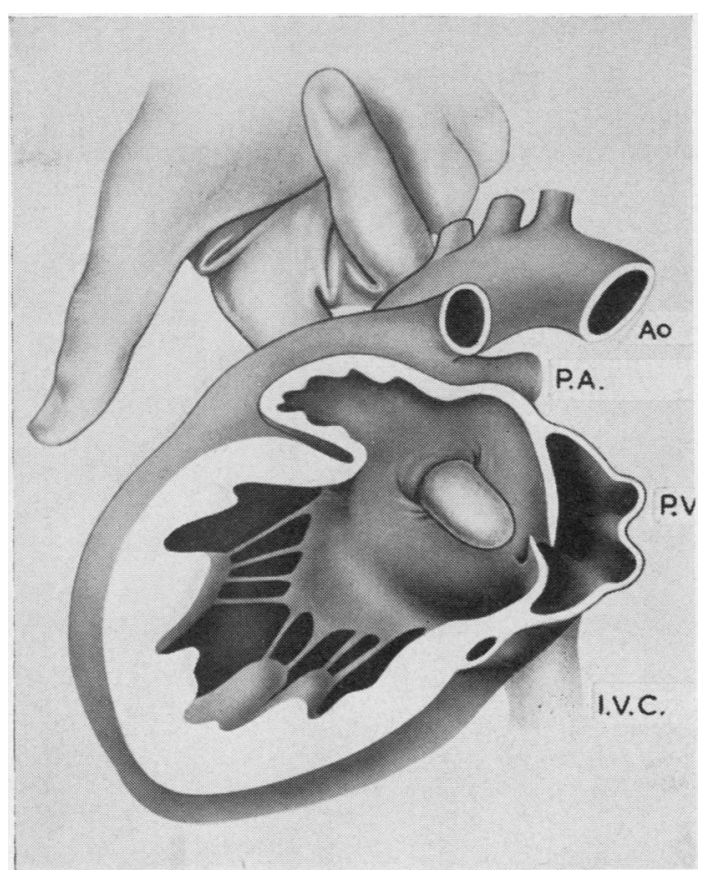

FIG. 6. Case 2. Palpation via the right atrium. The right index finger passes a small dorsal atrial septal defect of the central (secundum) type. The palpating finger enters the lower compartment and feels the mitral orifice and the abnormal septum. A.=aorta; $P . A .=$ pulmonary artery; P.V.= pulmonary veins; $I . V . C .=$ inferior vena cava .

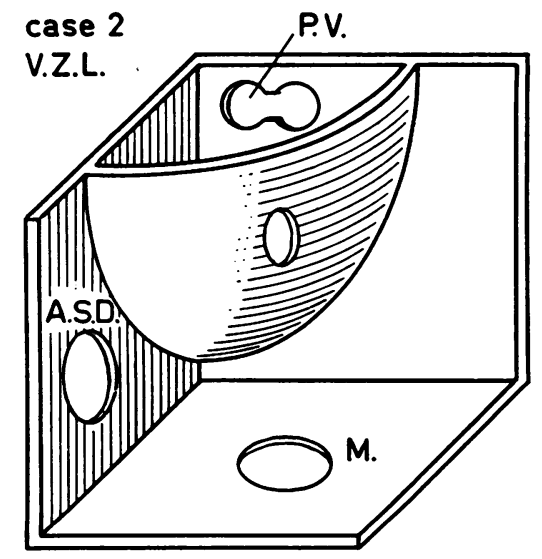

FIG. 7. Case 2. Diagram of the left atrium, divided into two compartments by an abnormal septum. The upper compartment receives the pulmonary veins (P.V.), while the lower communicates with the mitral orifice $(M$.$) . The$ abnormal septum shows an orifice of $0.5 \mathrm{sq}$. $\mathrm{cm}$. The lower compartment communicates with the right atrium via a small dorsal atrial septal defect (A.S.D.) of the central (secundum) type.

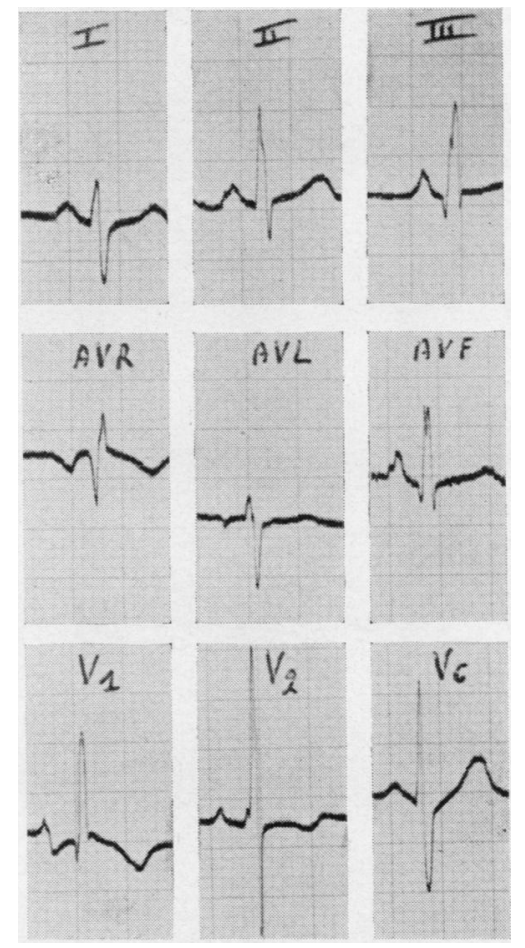

FIG. 8. Case 3. E.C.G. showing sinus rhythm, hypertrophy of the right ventricle, and strain; right bundle-branch block.

level. The pressure in the right ventricle was increased to $47 / 4 \mathrm{~mm}$. $\mathrm{Hg}$ and that in the pulmonary artery to $45 / 33 \mathrm{~mm}$. $\mathrm{Hg}$. The catheter failed to reach a peripheral site in the pulmonary artery, nor was it possible to enter the left atrium. The ratio between the pulmonary and systemic circulation was $3: 1$.

Atrial septal defect was diagnosed; the electroand vectorcardiogram indicated the dorsal (secundum) type. Auscultation and a phonocardiogram in addition suggested mild mitral stenosis. The pressure in the left atrium, however, was unknown.

In view of this diagnosis an operation was performed on 2 January 1963 via a bilateral submammary incision in the fifth intercostal space, using moderate hypothermia. Palpation in the right atrium disclosed a small dorsal atrial septal defect of the central (secundum) type, through which blood flowed with considerable force from left to right. Further palpation showed that the pulmonary veins of the right lung and a small vein of the left lung opened up into that part of the heart which was reached by passing the small central atrial septal defect. In this part of the heart, however, no mitral orifice was palpable. Palpation along the left auricle in the left atrium proved that the latter was very small and received the left pulmonary veins, except the pectoral branch, from the upper lobe. There was no com- 


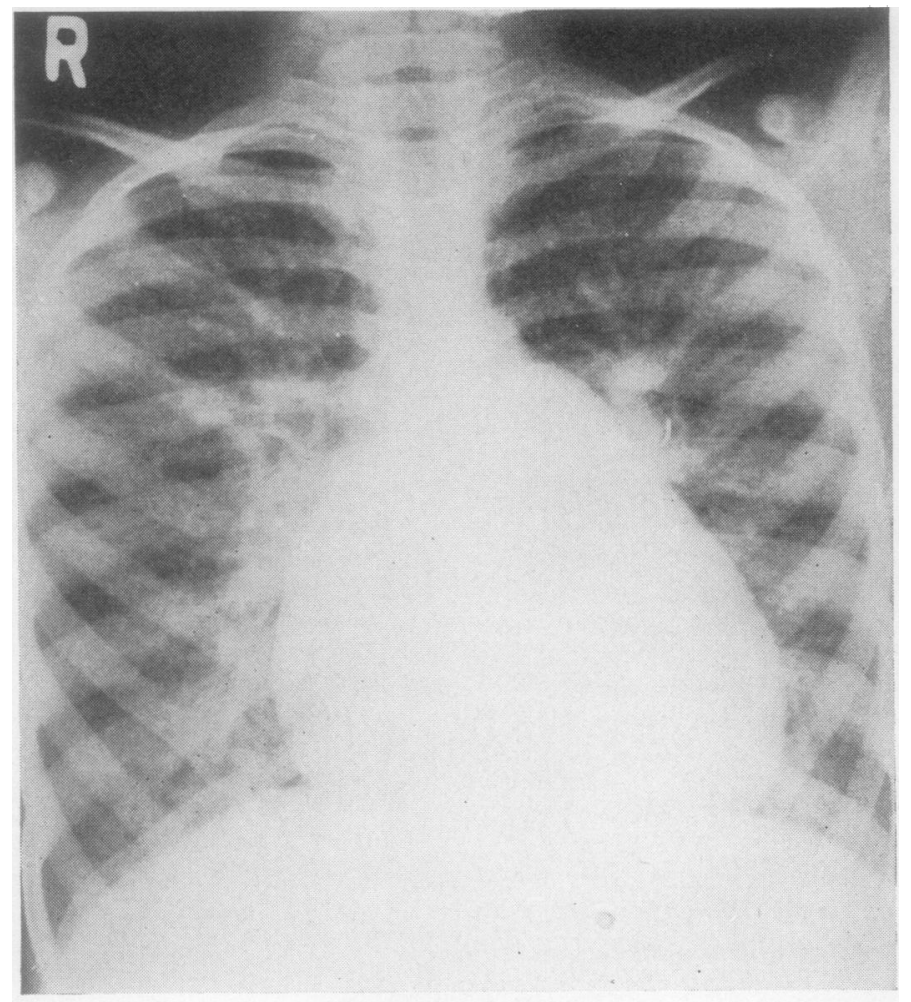

(a)

munication between the left atrium and the abovementioned compartment of the heart (Fig. 10).

The diagnosis of cor triatriatum was made preoperatively.

The circulation was interrupted and the right atrium was opened. The abnormal diaphragm separating the spatium pulmonale from the left atrium was palpated and resected through the atrial septal defect, using a grooved catheter left in the left atrium as a guide. The atrial septal defect was closed. After restoration of the circulation the oxygen samples no longer showed a shunt, and there was no longer a pressure gradient between the former spatium pulmonale and the left atrium.

This third patient was operated upon for an atrial septal defect of the dorsal (secundum) type with mild mitral stenosis and moderate pulmonary hypertension. This diagnosis had been based on the clinical findings, electrocardiogram, phonocardiogram and vectorcardiogram, radiographs, and angiocardiogram, which suggested an enlarged left atrium where in fact was the spatium pulmonale. Unfortunately, no wedge pressure was recorded in the pulmonary artery.

The operation was performed under moderate hypothermia. No communication existed between the

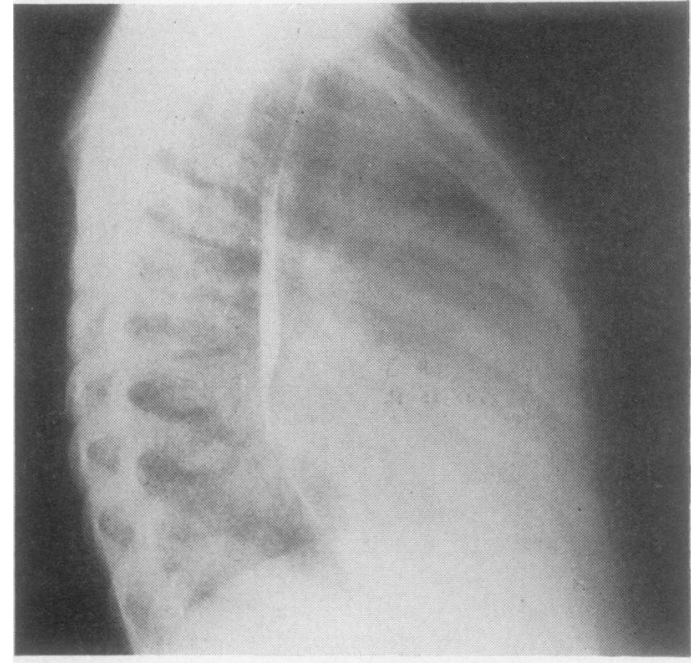

(b)

FIG. 9. Case 3. Chest radiographs in (a) antero-posterior and (b) lateral projections: pronounced pulmonary vascular pattern; enlarged right atrium and right ventricle. 


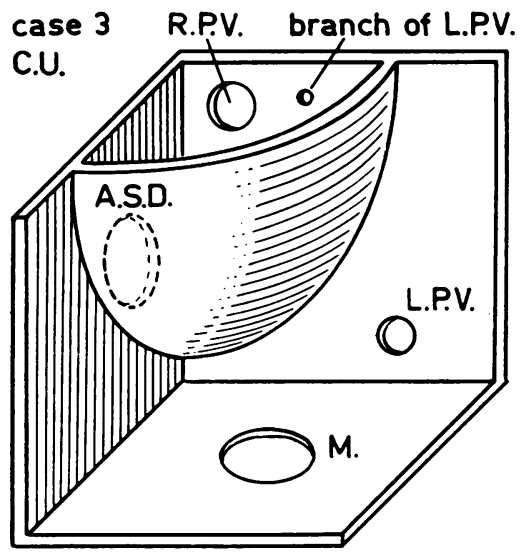

FIG. 10. Case 3. Diagram of the left atrium, divided into two compartments by an abnormal septum. The upper compartment receives the right pulmonary veins (R.P.V.) and $a$ branch of the left pulmonary veins. The lower compartment communicates with the mitral orifice (M.) and receives the left pulmonary veins (L.P.V.), with the exception of the pectoral branch, from the upper lobe. The abnormal septum has no orifice. The upper compartment communicates with the right atrium via a small central (secundum) atrial septal defect.

third atrium and the left atrium. The former received the right pulmonary veins and a small left pulmonary vein and communicated with the right atrium via a dorsal (secundum) atrial septal defect. The blood which passed the mitral valves originated exclusively from the left lung (Fig. 10).

CASE 4 S. H. was a man aged 37 who in 1963 was examined because for four months he had shown dyspnoea on exertion and experienced a stabbing sensation below the left costal margin, especially on exertion. Auscultation disclosed to the left of the sternum a grade 2 protosystolic souffle and a bifid second sound. No souffle was heard at the apex.

The chest radiograph showed a rather large heart with slightly broadened hila. In the left oblique lateral projection, both ventricles were slightly enlarged. The electrocardiogram showed right ventricular hypertrophy and dilatation, an incomplete right bundle-branch block, and left axis rotation; the vectorcardiogram was suggestive of an atrial septal defect of the ventral (primum) type.

This patient's condition was finally diagnosed as atrial septal defect of the dorsal (secundum) type ; since the symptoms had exacerbated and the heart was unmistakably enlarged, an operation was considered advisable.

The operation was performed on 13 September 1963 through an antero-lateral incision in the fifth right intercostal space with extracorporeal circulation. The right atrium was slightly dilated, and on palpa- tion a dorsal septal defect of the central (secundum) type was felt. Through this atrial septal defect the surgeon could enter either a compartment with the mitral valves or a compartment into which the pulmonary veins opened. The two compartments were separated by a septum with an orifice (Fig. 11).

The patient was connected to the heart-lung apparatus, and the right atrium was opened. The septum between the left atrium and the third atrium was partly resected, and the atrial septal defect was closed.

Oxygen samples no longer showed a left-to-right shunt at the atrial level. The various follow-ups disclosed a very satisfactory general condition; the heart had become smaller.

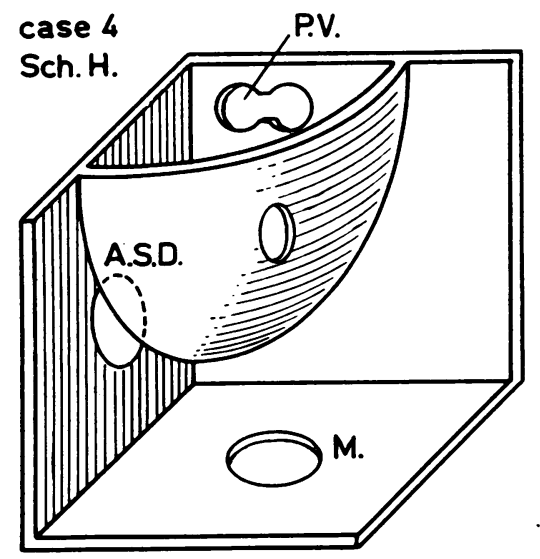

FIG. 11. Case 4. Diagram of the left atrium, divided into two compartments by an abnormal septum. The upper compartment receives the pulmonary veins $(P . V$.$) , while$ the lower communicates with the mitral orifice $(M$.$) . The$ abnormal septum has an orifice of $0.5 \mathrm{sq} . \mathrm{cm}$. The two compartments communicate with the right atrium via a central (secundum) atrial septal defect.

The diagnosis of cor triatriatum was established pre-operatively. The operation had been started with a diagnosis of atrial septal defect of the dorsal (secundum) type, although a ventral (primum) type could not be excluded with certainty in view of the electro- and vectorcardiogram findings. The operation was performed with extracorporeal circulation. There was an orifice connecting the left atrium with the third atrium. Via a dorsal (secundum) atrial septal defect both the left atrium and the third atrium communicated with the right atrium (Fig. 11).

\section{DISCUSSION}

CLASSIFICATION In 1949 Loeffler suggested a division into three groups, on the basis of the communication between the two compartments 
and their communication, if any, with the right atrium.

In the first group the abnormal diaphragm shows no orifice. This condition is incompatible with life unless there is an open foramen ovale and an atrial septal defect, or unless a proportion of the pulmonary veins communicate with the lower compartment.

In the second group the intra-atrial diaphragm has one or several orifices. These patients present the features of mitral stenosis when the interatrial septum is intact, or the features of a Lutembacher syndrome when the upper compartment communicates with the right atrium via an atrial septal defect.

The third group includes all cases in which the left atrium contains an incomplete band-shaped septum. These fibro-muscular bands never produce clinical symptoms, and we do not regard these patients as cases of cor triatriatum.

DEVELOPMENTAL CONSIDERATIONS As early as 1903 Griffith suggested that cor triatriatum might result from a fault at the junction of the pulmonary vein trunk to the left atrium. Much later this theory was revived by Pfennig (1941), Loeffler (1949), Parsons (1950), Edwards (1953), and Hartmann (1955).

The lungs and the primitive digestive tube originally share a common capillary venous system which has connexions with the umbilico-vitellin and the cardinal system and therefore drains into the right part of the atrium commune.

Secondarily, the dorsal wall of the left part of the atrium commune evaginates in the direction of the lungs. This evagination (the common pulmonary vein) forms connexions with the capillary plexus of the lungs, while the connexions with the umbilico-vitellin and the cardinal system are obliterated. At an even later stage the common pulmonary vein dilates to form the spatium pulmonale, into which the four pulmonary veins separately drain. This spatium is subsequently incorporated in the left atrium. The left atrium then consists of two parts-namely, the original part which communicates with the auricle and the mitral orifice, and the part formed by the spatium pulmonale.

In cor triatriatum the spatium pulmonale is probably not incorporated in the left atrium. The latter continues to consist of two parts, partly separated by a wall duplication which, on the left side, contains the left superior vena cava, reduced to Marshall's ligament (Los, 1958). In some cases, however, the two parts prove not to communicate. a condition which has so far remained unexplained (our Case 3).

The wall duplication, which, if it persists, constitutes the abnormal septum in cor triatriatum, consists of the posterior wall of the primitive atrium and the inferior wall of the spatium pulmonale.

Accepting the embryological explanation offered by Los (1958), we regard the third atrium in cor triatriatum as a persistent spatium pulmonale arising from a primordium which grows at its normal site. The right wall of this spatium develops into the primary septum with the fossa ovalis, while the left wall develops a duplication containing Marshall's ligament or a persistent left superior vena cava. Unlike Grondin, Leonard, Anderson, Amplatz, Edwards, and Varco (1964) we apply the designation cor triatriatum only if the above criterion is met.

To ensure a better embryological explanation of the anomalies seen, it will in future be necessary always to determine with accuracy the position of Marshall's ligament, or the foramen ovale (if it persists) will have to be determined.

In our Case 3 the pulmonary veins of the left lung opened into the left atrium, whereas those of the right lung drained into the third atrium. We can offer no embryological explanation for this. At least the position of Marshall's ligament or of the fossa ovalis should be known if such an explanation is to be found.

CLINICAL PICTURE AND DIAGNOSIS Cor triatriatum occurs in children as a congenital cardiopathy which causes little cyanosis, if any. It is associated with pronounced dyspnoea and marked enlargement of the right heart with overfilling of the lungs. Auscultation discloses a systolic or presystolic and systolic souffle. The electrocardiogram reveals an isolated hypertrophy of the right heart, and the radiographs show an abnormal bulge above and behind the left atrium.

In adults the clinical, radiological, and electrocardiographic features of this anomaly are reminiscent of a narrow mitral stenosis with pulmonary hypertension.

A definite diagnosis can be made only on the basis of a pressure gradient on both sides of the abnormal diaphragm or on the basis of an angiocardiographic indication of a compartment into which the pulmonary veins drain.

The differential diagnosis in children must consider an atrial septal defect with pulmonary hypertension (enlarged heart and overfilled lungs). The so-called Lutembacher's syndrome must also be taken into account. Finally, cor triatriatum can 
also be simulated by total abnormal venous drainage into the right atrium. In Lutembacher's syndrome, however, there is a tendency toward equalization of the pressures in the right and left atria so that the capillary pulmonary pressure is either normal or only slightly increased.

In adults we must differentiate from mitral stenosis with pulmonary hypertension and from primary pulmonary hypertension. In the latter case heart catheterization affords proof of the precapillary origin of the pulmonary hypertension (which in the case of cor triatriatum is postcapillary).

\section{SUMMARY}

Four cases of cor triatriatum are discussed. The diagnosis was established pre-operatively in two patients. Surgical treatment was successful in all patients. In Case 3 the left pulmonary veins drained into the left atrium, while the right pulmonary veins opened into the third atrium. Embryologically this condition cannot be explained merely by a persistent spatium pulmonale.

\section{REFERENCES}

Abbott, M. E. (1946). In Nelson Loose-Leaf Medicine, vol. 4, p. 207. Nelson, New York.

Barnes, C. G., and Finlay, H. V. L. (1952). Cor triatriatum. Brit. Heart J., 14, 283.

Barrett, N. R., and Hickie, J. B. (1957). Cor triatrium. Thorax, $12,24$.

Edwards, J. E. (1953). Pathologic and developmental considerations in anomalous pulmonary venous connection. Proc. Mayo Clin., 28, 441.

Griffith, T. W. (1903). Note on a second example of division of the cavity of the left auricle into two compartments by a fibrous band. J. Anat. (Lond.) Physiol., 37, 255.

Grondin, C., Leonard, A. S., Anderson, R. C., Amplatz, K. A., Edwards, J. E., and Varco, R. L. (1964). Cor triatriatum: a
diagnostic surgical enigma. J. thorac. cardiovasc. Surg., 48, 527.

Hartmann, H. A. (1955). Cor triatriatum (stenosis of common pulmonary vein). Arch.Path., 59, 706.

Lewis, F. J., Varco, R. L., Taufic, M., and Niazi, S. A. (1956). Direct vision repair of triatrial heart and total anomalous pulmonary venous drainage. Surg. Gynec. Obstet., 102, 713.

Loeffler, E. (1949). Unusual malformation of the left atrium: pulmonary sinus. Arch. Path., 48, 371.

Los, J. A. (1958). De embryonale ontwikkeling van de venae pulmonales en de sinus coronarius bij de mens (with an English summary). Diss, Leiden.

Niwayama, G. (1960). Cor triatriatum. Amer. Heart J., 59: 291.

Parsons, C. G. (1950). Cor triatriatum. Brit. Heart J., 12, 327.

Pfennig, E. (1941). Anomale Septumbildung im linken Vorhof des menschlichen Herzens. Virchows Arch. Path. Anat., 307, 579.

Vineberg, A., and Gialloreto, O. (1956). Report of a successful operation for stenosis of common pulmonary vein (cor triatriatum). Canad. med. Ass. J., 74, 719 . 